\title{
The Effects of Traditional Hand written and Web-Based Questionnaires on Clinical Research: Using UDI-6, IIQ-7, and PISQ-12 as Examples
}

\author{
Shiaw-Wen Tien ${ }^{1}$, Hsiu-Lin Chen ${ }^{2, *}$ and Chia-Hsiang Hsieh ${ }^{3}$
}

\author{
${ }^{I}$ Department of Business Administration, Chung Hua University, 707, Sec. 2, WuFu Rd., Hsinchu 30012, Taiwan ROC; \\ ${ }^{2}$ Ph.D. Program of Technology Management, Chung Hua University, 707, Sec. 2, WuFu Rd., Hsinchu 30012, Taiwan \\ ROC; ${ }^{3}$ Department of Marketing, Kainan University, No. 1 Kainan Road, Luzhu Shiang, Taoyuan 33857, Taiwan ROC
}

\begin{abstract}
Web-based surveys are frequently applied in clinical research. In this study, hospital patients were requested to complete web-based and traditional hand written questionnaires. The t test and intraclass correlation coefficients were used to compare the results of the web-based and traditional hand written questionnaires. The survey results of 81 participants showed no significant score differences between the web-based and traditional hand written questionnaires.

The 81 participants did not show any preference for either web-based or pencil-and-paper based surveys, and the statistical results showed no significant difference between the two types of survey. Therefore, this study suggested using webbased questionnaires instead of pencil-and-paper based questionnaires, because using web-based questionnaires can decrease doctor consultation time, reduce labor costs, provide patients with remote self-assessment services prior to a clinic visit and adequately collect questionnaires to enhance research capability. Furthermore, this type of questionnaire survey enables patients to answer personal questions honestly.
\end{abstract}

Keywords: Incontinence impact questionnaire-7 (IIQ-7), Pelvic organ prolapse/urinary incontinence sexual function questionnaire-12 (PISQ-12), Pencil-and-paper questionnaire, Urinary distress inventory-6 (UDI-6), Urinary incontinence, Web-based questionnaire.

\section{INTRODUCTION}

Currently, high information accessibility and improved technology related to memory bandwidth have rendered computers and the Internet as necessary elements in daily life. In recent years, numerous studies have investigated the reliability and validity of web-based surveys and internet characteristics that affect survey results. Research findings have shown that web-based questionnaire surveys are as reliable as interview surveys [1], that the personal characteristics and ethnicity of interviewees influence interview results [2, 3], and that internet problems such as bandwidth also affect survey quality [4]. Designs, such as questionnaire order, directory labels and graphical display, can enhance the quality of web-based questionnaires [5]. The relationship between interviewers and interviewees and the time required to answer a questionnaire can affect the degree to which interviewees cooperate $[6,7]$. Therefore, studies concentrated on the effect of changes in information technology for information collection $[8,9]$.

Since web-based surveys are not limited by time and space, they have attracted substantial attention and have been applied in clinical trials and epidemiology studies. Surveys on the quality of life of patients have involved using webbased approaches as feature management [10]; however,

*Address correspondence to this author at the School of Ph.D. Program of Technology Management, Chung Hua University, 707, Sec. 2, WuFu Rd., Hsinchu 30012, Taiwan ROC; Tel: 0963320344;

E-mail: s210@eip.mkc.edu.tw whether the effects of such approach are equivalent to those observed in traditional questionnaire surveys depends on the survey content. Regarding quality-of-life surveys involving people with irritable bowel syndrome, gastro esophageal reflux, indigestion, chronic diseases, and arthritis, no difference was found between web-based and traditional questionnaire surveys [11]. For sensitive topics or behaviors such as sex life, questionnaire models considerably affect the return rates and validity of the questionnaire [12]. This study selected patients with urinary incontinence as research participants, investigating whether survey results of Web-based urinary distress inventory-6 (UDI-6), incontinence impact questionnaire-7 (IIQ-7), and Pelvic Organ Prolapse/Urinary Incontinence Sexual Function Questionnaire-12 (PISQ-12) are consistent with those attained using the traditional hand written questionnaires.

Female urinary incontinence is often inevitable during aging. Approximately one third of women experience urinary incontinence and the prevalence of which increases with age. Previous studies have shown that diabetes, high blood pressure, drug allergy, obesity, smoking, hormone therapy, gynecological surgery and hysterectomy are risk factors for urinary incontinence in elderly Taiwanese women and aging is a critical risk factor for urinary incontinence. In addition, UDI-6, IIQ-7 and PISQ-12 are extremely helpful for doctors when assessing patients with urinary incontinence during urinary bladder and voiding dysfunction treatments. As patients typically consider their conditions to be extremely severe, doctors can objectively assess the patient's 
condition by using these questionnaires. UDI-6 and IIQ-7 assess the severity of urinary incontinence and the quality of patient life; high scores indicate severe urinary incontinence and adverse quality of life. PISQ-12 assesses the quality of sex life of women, where a high score indicates excellent sexual functions [13].

For clinical research that involves questionnaire surveys, using pencil-and-paper questionnaires may yield invalid results because of incomplete responses, illegible writing and noncompliance. In addition, human errors occur during data entry. By contrast, web-based questionnaires provide immediate feedback to interviewers for data input and mitigate data entry errors; therefore, the required data can be rapidly and accurately collected for data analysis. In addition to eliminating the printing and labor costs required for traditional hand written surveys, web questionnaire surveys can enhance the accuracy of the collected data. Since 1990, several computer-aided self-interviewing systems [14] have been developed for interviewees to complete the questionnaires using computers, thereby improving the shortcomings of pencil-and-paper based surveys.

Due to insufficient medical knowledge, patients do not completely understand the association of a questionnaire to their diseases; thus, they may provide incomplete answers when questions involve personal or embarrassing matters. Thus, this study endeavors to understand: (a) whether the types of questionnaires used affect interviewees' willingness to answer questions; (b) whether the types of questionnaires used influence the integrity of questionnaires; (c) whether suitable methods are available for questionnaires of various attributes; (d) whether demographic factors (including age, area of residence, education level and computer experience) and the types of questionnaires used influence result consistency; and (e) whether web-based or traditional hand writtenquestionnaires can serve as a self-assessment tool before a clinical visit.

\section{METHODS}

This study selected female patients with urinary incontinence, urinary leakage and urinary urgency from an obstetrics and gynecology clinic that specializes in female urinary incontinence at a first-class medical center in Taipei. Over a period of 1 year, patients were recruited regardless of their age, education level and area of residence, and were informed of the study objectives. Upon receiving their oral consent, the patients were instructed to complete pencil-andpaper and web-based questionnaires during two separate visits to the clinic. Taipei is a densely populated area with convenient transportation and the medical center treats patients with various backgrounds; thus, diverse data were obtained from this medical center.

With support from doctors, this study systematically administered the questionnaires to the patients. A urinaryincontinence and pelvic-prolapse therapy center website was established to introduce related examination equipment, items, precautions and medical knowledge. In addition, webbased questionnaires UDI-6, IIQ-7, and PISQ-12 were constructed. When the patients would visit the clinic, they would complete a pencil-and-paper questionnaire. Subsequently, they would answer the web-based questionnaire the follow- ing week before receiving the physiological examinations. The traditional hand written and web-based questionnaires with complete responses were adopted for data analysis.

This study employed a random sampling design and did not screen patients in advance. The patients completed traditional hand written and web-based questionnaires with a 1week interval. The participants used their national identification codes as their password for entering the web-based survey system. The survey duration was 30 minutes. Following the web-based survey, a physiological examination procedure was commenced in which the examined items included urinary flow rate, urinary bladder volume and pressure, electromyography, urethral pressure and video urodynamics. The content and questions of the pencil-and-paper and web-based questionnaires were identical, except that the beginning of the web-based questionnaire included questions about computer experience and education level.

After approximately 1 year, 81 patients completed the questionnaires. The total scores for each type of questionnaire and the scores for each question item were obtained. $\mathrm{T}$ tests and intraclass correlation coefficients (ICCs) were used to assess the collected data. The ICCs for both the penciland-paper and web-based surveys exceeded $0.99(p<0.05)$.

\section{RESULTS}

In this study, 91 patients from an obstetrics and gynecology clinic that specializes in female urinary incontinence at a medical center were invited to participate in this study. Out of the 91 patients, 81 patients consented to participate and completed the pencil-and-paper and web-based versions of UDI-6, IIQ-7 and PISQ-12. These questionnaires were completed in a random order. The completion rate and integrity of the pencil-and-paper version of the three questionnaires approximated those of the web-based version. However, the completion rate and integrity of PISQ-12, which involves personal privacy, were below $70 \%$.

The results showed no significant difference between the traditional hand written and web-based versions of UDI-6, IIQ-7, and PISQ-12 in terms of total scores and the scores for each question item.

This study also investigated whether patients' background affects their responses to different types of medical questionnaires. Therefore, the design of the web-based questionnaires excluded any program verification procedure that would prohibit patients from skipping questions. Similar to traditional hand written questionnaires, the Web-based questionnaires allowed respondents to leave the questions blank. However, no illegible responses occurred for web-based questionnaires.

Firstly, this study compared the completion rate (i.e., leaving the entire questionnaire blank) and the integrity rate (i.e., the response rate for a single questionnaire) of the traditional hand written questionnaire with those of the web version. As shown in Table 1, the completion rates and integrity rates of UDI-6 and IIQ-7 were higher than 95\%; specifically, the completion rates reached $100 \%$. However, both the completion rate and integrity rate of PISQ-12 were approximately $70 \%$. Out of 27 women with low PISQ-12 completion rates and integrity scores, the education levels of 15 women 
Table 1. The completion rates and integrity rates of various questionnaires.

\begin{tabular}{|c|c|c|c|c|c|c|}
\hline \multirow{2}{*}{ Item } & \multicolumn{2}{|c|}{ UDI-6 } & \multicolumn{2}{c|}{ IIQ-7 } & \multicolumn{2}{c|}{ PISQ-12 } \\
\cline { 2 - 7 } & Completion Rate & Integrity Rate & Completion Rate & Integrity Rate & Completion Rate & Integrity Rate \\
\hline \hline Web & $100 \%$ & $99.37 \%$ & $100 \%$ & $99.65 \%$ & $72 \%$ & $72.75 \%$ \\
\hline Pencil and paper & $95 \%$ & $96.09 \%$ & $95 \%$ & $95.06 \%$ & $67 \%$ & $67.59 \%$ \\
\hline
\end{tabular}

Table 2. The differences between web-based and pencil-and-paper questionnaires for participants with various backgrounds.

\begin{tabular}{|c|c|c|c|}
\hline$(\mathrm{n}=81)$ & UDI-6 & IIQ-7 & PISQ-12 \\
\hline$<40$ & 0.202 & 0.482 & 0.396 \\
\hline $41 \sim 50$ & $0.018^{*}$ & 0.076 & 0.251 \\
\hline$>60$ & 0.432 & NA & NA \\
\hline \multicolumn{4}{|l|}{ Education (mean, SD) } \\
\hline None & 0.070 & 0.374 & 0.391 \\
\hline College or graduate degree & $0.012 *$ & 0.212 & 0.436 \\
\hline \multicolumn{4}{|l|}{ Residence (mean, SD) } \\
\hline Taipei City & $0.050^{*}$ & 0.068 & 0.113 \\
\hline Taipei County & $0.044^{*}$ & 0.285 & 0.620 \\
\hline Another & 0.636 & 0.259 & NA \\
\hline \multicolumn{4}{|l|}{ Computer experience (mean, SD) } \\
\hline None & 0.447 & 0.711 & 0.266 \\
\hline
\end{tabular}

$* \mathrm{p}>0.05$

were below junior high school, their average age was 58 years and their computer experience averaged to 3 years at most.

Secondly, based on the overall UDI-6, IIQ-7, and PISQ12 scores, this study investigated whether different questionnaire types yielded consistent results for patients with various backgrounds (including age, residence place, education level, and computer experience). The research results are presented in Table 2.

A significant difference was found between the traditional hand written and web-based UDI- 6 completed by the $41-$ to 50-year-old age group; for other age groups, no significant difference was observed $(p=0.018)$. Considering education level, only among the patients whose education levels were above senior high school occurred a significant difference between the two versions of UDI-6. Considering area of residence, a significant difference existed between the two versions of UDI-6 for the Taipei and New Taipei City groups. The group with more than 10 years of computer experience exhibited a significant difference in their traditional hand written and web-based UDI-6 results.

Finally, to address whether a web-based or pencil-andpaper based questionnaire can serve as a self-assessment 
auxiliary tool before a clinical visit, this study examined the correlation between the physiological results of urine leakage and the two versions of the questionnaires. As shown in Table 3, the scores of UDI-6 and IIQ-7 were highly correlated with urine leakage. Particularly, the correlation coefficients for both traditional hand written and web-based UDI-6 exceeded 0.4 and the PISQ-12 scores were negatively correlated with urine leakage.

Table 3. The intraclass correlations between urine leakage and the web-based and pencil-and-paper based survey results.

\begin{tabular}{|c|c|c|}
\hline & R Web (P Value) & R Paper (P Value) \\
\hline \hline UDI-6 & $0.414(0.000)$ & $0.459(0.000)$ \\
\hline IIQ-7 & $0.244(0.036)$ & $0.255(0.028)$ \\
\hline PISQ-12 & $-0.013(0.911)$ & $-0.040(0.783)$ \\
\hline
\end{tabular}

\section{CONCLUSION}

In this study, it was found that the web-based and traditional hand written surveys were equally acceptable for the participants but most participants preferred the web-based UDI-6, IIQ-7 and PISQ-12 surveys. For questions involving personal privacy, the web-based survey was found to be more acceptable for participants than the pencil-and-paper based survey. As shown in Table 1, the completion rates and integrity rates of UDI-6 and IIQ-7 were higher than those of PISQ-12 as UDI-6 and IIQ-7 involved urinary incontinence and entailed less embarrassing items and questions related to personal matters.

In summation, the difference in questionnaire types did not affect the patients' willingness to complete medical questionnaires. However, the completion rates and integrity rates of the web-based questionnaires were higher than those of the traditional hand written versions. For various types of questionnaires, responses to the web-based and traditional hand written versions of questionnaires were consistent. According to statistical results, only a small portion of participants with differing backgrounds (age, education, and residential area) who completed the Web and traditional hand written questionnaires exhibited a significant difference in their results $(\mathrm{p}<0.05)$.

Therefore, different versions of questionnaires did not yield inconsistent results among participants of varying backgrounds. The ICC results indicated that the pencil-andpaper and web-based surveys and urine leakage were strongly correlated; therefore, web-based or traditional hand written questionnaires can be used as a self-assessment auxiliary tool before a clinical visit.

Although no difference existed between the applications of the two types of surveys to clinical research, previous studies have indicated that web-based surveys are extremely useful for example, these surveys possess excellent integration ability, can process complex questionnaire analysis, reduce errors and manage data that require special treatment $[2,15]$. In addition, electronic questionnaires can be designed to automatically time-stamp the data [14], thereby accurately reflecting the time at which a questionnaire is completed. On-screen questionnaire formats can reduce the content, thus eliminating the factors that affect participants' intention to complete the questionnaires. Other studies have shown that $5 \%-10 \%$ of respondents skipped question items while completing pencil-and-paper questionnaires [14]. In addition, the omission rate increased as the length of a questionnaire increased [16]. Web-based questionnaires are beneficial because interviewees cannot skip question items on web-based questionnaires; they can eliminate the use of excessively long questionnaire formats, prohibit respondents from skipping questions, or easily reuse questions in another questionnaire survey.

The researchers of this study speculated that age, education level, area of residence and computer experience affect the survey results. However, no significant difference was found. Moreover, only a small number of participants in this study had higher education levels; therefore, this study could not extensively examine differences. Nevertheless, other studies have shown that factors like age, education level and computer experience do not significantly affect the electronic survey results $[2,15]$.

\section{FURTHER ANALYSIS}

Small sample size is a limitation in this study. The participants were a random sample, which is probably not representative. Due to high information accessibility and universal access to education, an increasing number of people are highly educated and familiar with computers. According to this study, no difference was found between the clinical applications of pencil-and-paper and web-based questionnaires. Therefore, this study recommends that future studies should focus on web-based questionnaire designs and investigate the perspectives of interviewees using web-based questionnaires, thereby facilitating the extensive application of webbased questionnaires in clinical research.

\section{CONFLICT OF INTEREST}

The authors confirm that this article content has no conflict of interest.

\section{ACKNOWLEDGEMENTS}

Declared none.

\section{REFERENCES}

[1] R.S. Burt, "Network items and the general social survey", Social Networks, vol. 6, pp. 293-339, 1984.

[2] T. Kogovšek, and A. Ferligoj, "Effects on reliability and validity of egocentered network measurements", Social Networks, vol. 27, pp. 205-229, 2005.

[3] A. Marin, and K. N. Hampton, "Simplifying the personal network name generator: alternatives to traditional multiple and single name generators", Field Methods, vol. 19, pp. 163-193, 2007.

[4] C. M. Carty, P.D. Killworth, H.R. Bernard, E.C. Johnsen, and G.A. Shelley, "Comparing two methods for estimating network size", Human Organization, vol. 60, pp. 28-39, 2001.

[5] D.M. Bushnell, M. C. Reilly, C. Galani. J.F. Ricci, M.L. Martin D.L. Patrick, and C. McBurney, "Validation of electronic data capture of the irritable bowel syndrome-quality of life measure, the work productivity and activity impairment questionnaire for irritable bowel syndrome and the euroQol", Value Health, vol. 9, no. 2, pp. 98-105, 2006. 
[6] P.V. Marsden, and K.E. Campbell, "Measuring ties strength", Social Forces, vol. 63, pp. 482-501, 1984.

[7] C. M. Carty, and A. Wutich, "Conceptual and empirical arguments for including or excluding ego from structural analyses of personal networks", Connections, vol. 26, pp. 82-88, 2005.

[8] R.G. Rogers, K.W. Coates, D. K. Doak, S. Khalsa, C. Qualls, "A short form of the pelvic organ prolapse/urinary incontinence sexual questionnaire (PISQ-12)", International Urogynecology Journal and Pelvic Floor Dysfunction, vol. 14, pp. 164-8, 2003.

[9] K.J.S. Hoo, How Much is Enough? A Risk-Management Approach to Computer Security, Consortium for Research on Information Security and Policy (CRISP) Stanford University, 2000.

[10] H.A. Bischoff-Ferrari, M. Vondechend, N. Bellamy, and R. Theiler, "Validation and patient acceptance of a computer touch screen version of the WOMAC 3.1 osteoarthritis index", Annuals of Rheumatic Diseases, vol. 64, no. 1, pp. 80-84, 2005.

[11] S. Hansche, "Designing a security awareness program: Part 1, information", Information Systems Security, vol. 9, no. 6, pp. 14-22, 2001

[12] L. Kleinman, N.K. Leidy, J. Crawley, A. Bonomi, and P. Schoenfeld, "A comparative trial of paper-and-pencil versus computer admin- istration of the Quality of Life in Reflux and Dyspepsia (QOLRAD) questionnaire", Medical Care, vol. 39, no. 2, pp. 181$189,2001$.

[13] R. Tourangeau, and T. Smith, "Asking sensitive questions: the impact of data collection mode, question format, and question context”, Public Opinion Quarterly, vol. 60, no. 2, pp. 275-304, 2006.

[14] J.S. Uebersax, J.F. Wyman, S.A. Shumaker, D.K. M. Clish, J.A. Fantl, "Short forms to assess life quality and symptom distress for urinary incontinence in women: The incontinence impact questionnaire and the urogenital distress inventory", Continence Program for Women Research Group Neurourol Urodyn, vol. 14, pp. 131139, 1995.

[15] T. Kogovšek, A. Ferligoj, W.S. Saris, and G. Coenders, "Estimating the reliability and validity of personal support measures: full information ML estimation with planned incomplete data", Social Networks, vol. 24, pp. 1-20, 2002.

[16] H. R. Bernard, E.C. Johnsen, P. D. Killworth, C. M. Carty, G.A. Shelley, and S. Robinson, "Comparing four different methods for measuring personal social networks", Social Networks, vol. 12, pp. $179-215,1990$

Received: September 16, 2014

Revised: December 23, 2014

Accepted: December 31, 2014

(C) Tien et al.; Licensee Bentham Open.

This is an open access article licensed under the terms of the Creative Commons Attribution Non-Commercial License (http://creativecommons.org/licenses/by-nc/4.0/) which permits unrestricted, non-commercial use, distribution and reproduction in any medium, provided the work is properly cited. 